\title{
Quality control and evaluation of Inonotus obliquus using HPLC method with novel marker compounds
}

\author{
Jin Hyeok Kim', Seol Kyu Park', Chong Woon Cho', Ba Vinh Le ${ }^{1,2}$, Young Ho Kim¹, Haiying Bao ${ }^{3}$,
} Kyung Tae Kim${ }^{4 *}$ and Jong Seong Kang ${ }^{1 *}$ (D)

\begin{abstract}
Current quality control of Inonotus obliquus requires chromogen complex content limit of $10 \%$ in accordance with the State Pharmacopoeia of the Union of Soviet Socialist Republics. However, this causes ambiguous results, impeding precise quality control. To improve ambiguous quality control criteria, this study developed a new HPLC method using two novel marker compounds (inotodiol and 33-hydroxylanosta-8,24-dien-21-al) to control the quality control of I. obliquus. The HPLC analysis was carried out in a C18 column with an isocratic elution of 95\% acetonitrile at $210 \mathrm{~nm}$. The developed method validated in terms of linearity, precision, accuracy, and recovery. The content criteria were established by the linear regression method and relative standard deviation method. As the results of the quantitative monitoring, $1.165 \mathrm{mg} / \mathrm{g}$ of inotodiol and $1.717 \mathrm{mg} / \mathrm{g}$ of 33-hydroxylanosta-8,24-dien-21-al, calculated by the relative standard deviation method, were suggested new quality criteria. A new HPLC method was developed to improve current quality control of I. obliquus and new lower content criteria were proposed as a result of quantitative monitoring.
\end{abstract}

Keywords: Inonotus obliquus, Inotodiol, 33-Hydroxylanosta-8,24-dien-21-al, HPLC, Quality control

\section{Introduction}

Mushrooms are a representative functional food because they contain many bioactive compounds, such as $\beta$ glucans, peptides, chitinous, terpenes, sterols, and phenolic compounds (Bach et al. 2017; Le et al. 2017; Yan et al. 2018). Among mushroom species, Inonotus obliquus has received much interest recently and its extract is sold as a functional food in markets in Korea and other Asian countries.

Classified in the Hymenochaetaceae family, I. obliquus is a type of white-rot fungus that can form a sclerotium

\footnotetext{
*Correspondence: kimkkt@deu.ac.kr; kangjss@cnu.ac.kr

The English in this document has been checked by at least two professional editors, both native speakers of English. For a certificate, please see: http:// www.textcheck.com/certificate/PcyoKV

${ }^{4}$ Department of Food Science and Technology, Dong-Eui University, Busan 47340 , Korea

${ }^{1}$ College of Pharmacy, Chungnam National University, Daejeon 34134, Korea Full list of author information is available at the end of the article
}

(Sun et al. 2008). It has a wedge-like shape and the surface is irregularly cracked, with skin that can split and peel off into pieces of $5 \sim 40 \mathrm{~cm}$ (Zheng et al. 2010). It is distributed in Russia, China, Europe, and North America, and is a well-known precious medicinal mushroom (Moon and Lee 2009) that is used to treat cancer and heart, liver, and stomach diseases (Xu et al. 2016).

Many studies of I. obliquus have been conducted to investigate its bioactive components (Shikov et al. 2014). The representative bioactive components of I. obliquus are polyphenols and triterpenoids, which have antitumor properties, and polysaccharides, which have anticancer and anti-diabetes properties (Tian et al. 2017; Wang et al., 2017a; Wang et al., 2017b; Xu et al. 2016). Due to its extensive bioactivity, the import volume of $I$. obliquus as a food item is increasing rapidly, having doubled between 2012 and 2016 (MFDS 2013, 2017). 
Currently, the quality control of I. obliquus is regulated by the State Pharmacopoeia of the Union of Soviet Socialist Republics (11th edition), with a quality criterion of a lower threshold of $10 \%$ chromogen complex (Medizina 1990). Chromogen complex is defined as substances that can be easily converted into a dye or other colored compounds, such as $\beta$-glucans, polyphenols, steroids, superoxide dismutases, minerals, and tyrosine (Oxford University Press 2010); it is used to visualize barely detectable compounds by visible light (Aguiar et al. 2003). It has been used as a marker for cells or tissues that need to be visualized when taking photographs (Panarelli et al. 2012). Furthermore, chromogen can be used as a marker to evaluate digestibility by quantifying chromogen levels using a spectrophotometer, which detects a compound using visible light absorption (Davidson 1954). However, it is not required to use markers with visible light absorption, as instruments using ultraviolet (UV) have been developed such as UV detectors, which can analyze compounds without visible absorption. Therefore, the chromogen should not be selected as a marker. Chromogen complex consists of varying contents of numerous compounds. Therefore, the total quantity of chromogen complex is difficult to control precisely when attempting to ensure the quality of $I$. obliquus. In modern pharmaceutical analysis, the use of the chromogen complex yields ambiguous results, and it is not a suitable marker for quality control. Nevertheless, there have been no investigations of potential new markers to replace the chromogen complex for the quality control of I. obliquus since the Russian regulations were published (Shikov et al. 2014).

In modern analytical chemistry, especially in the pharmaceutical industry, bioactive compounds are used as markers to determine the relationship between biological or pharmaceutical activity with the quantitative analytical results of bioactive compounds in quality control procedures. I. obliquus has well-known anti-cancer properties; therefore, some compounds with demonstrable anti- cancer effects are worth considering as novel marker compounds. The identification of new markers for the quality control of I. obliquus can be achieved through studies of pharmacological activity. Hexane and chloroform extracts of I. obliquus have been reported to have anti-cancer effects (Chung et al. 2010; Nakata et al. 2007). Furthermore, in our previous study, a dichloromethane extract of $I$. obliquus displayed an immunoregulatory effect related to anti-cancer activity, with the key compounds being inotodiol and 33-hydroxylanosta-8,24-dien-21-al, which were isolated from the dichloromethane extract of I. obliquus (Nguyet et al. 2018). Therefore, inotodiol and 3 $\beta$ hydroxylanosta-8,24-dien-21-al have the potential to be novel marker compounds for the quality control of $I$. obliquus due to their anti-cancer properties.

There have been few reports of the analysis of inotodiol obtained from I. obliquus (Du et al. 2011; Ju et al. 2010). Several reported analytical methods have been developed to verify the purity of extracted inotodiol and trametenolic acid and to detect phenolic acids and chromogen complex; however, these methods have not been validated and are not appropriate for the quality control of $I$. obliquus. Thus, new marker compounds and a new validated analytical method are required for the accurate quality control of I. obliquus. Therefore, the aim of this study was to develop an efficient and validated modern analytical method with novel marker compounds, including the optimization of extraction conditions, for the quality control and confirmatory testing of I. obliquus mushrooms.

\section{Materials and methods}

\section{Reagents and chemicals}

Twenty-five samples were obtained from a medicinal herb market in Korea and from Professor Haiying Bao of Jilin Agricultural University, China. The details of the samples are given in Table S1 and FigureS1. Inotodiol and 3ßhydroxylanosta-8,24-dien-21-al were isolated and confirmed using NMR analysis by professor Young Ho Kim

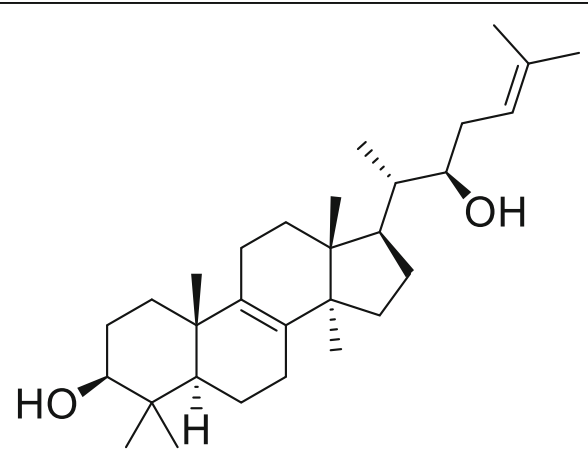<smiles>CC(C)=CCC[C@H](C=O)[C@H]1CC[C@]2(C)C3=C(CC[C@]12C)[C@@]1(C)CC[C@H](O)C(C)(C)C1CC3</smiles>

inotodiol

3ß-hydroxylanosta-8,24-dien-21-al

Fig. 1 Chemical structure of inotodiol and 3ß-hydroxylanosta-8,24-dien-21-al 
of Chungnam National University, Korea. The structures of the two isolated marker compounds are given in Fig. 1 and their high-performance liquid chromatography (HPLC) chromatograms are shown in Figure S2. HPLC grade methyl alcohol, acetonitrile, acetone, chloroform, dichloromethane, ethyl acetate, and hexane were purchased from Burdick and Jackson (Muskegon, MI, USA). Water was purified using a Milli-Q system (Milford, MA, USA).

\section{Standard and sample preparation}

Stock standard solutions $(1.0 \mathrm{mg} / \mathrm{mL})$ were prepared in methanol and stored in glass vials at $2{ }^{\circ} \mathrm{C}$. Each sample was pulverized and stored at $2{ }^{\circ} \mathrm{C}$. A 0.2 -g portion of pulverized sample was mixed with $20 \mathrm{~mL}$ of chloroform in a $50-\mathrm{mL}$ polypropylene conical tube from Corning Science Mexico (Reynosa, Tamaulipas, Mexico). Each sample solution was extracted by sonication $(40 \mathrm{kHz}$ and $400 \mathrm{~W}$ ) for $30 \mathrm{~min}$ using an SD-D250H sonicator from Sungdong (Seoul, Korea). The liquid phase of each sample was separated by filtration and completely evaporated at $50^{\circ} \mathrm{C}$ using an Eyela N-1000 rotary evaporator (Kyoto, Kyoto, Japan). Then, $2 \mathrm{~mL}$ of methyl alcohol was added and mixed using a Vortex Genie-2 mixer bade by Scientific Industries (Bohemia, NY, USA). All solutions were filtered through a $0.22-\mu \mathrm{m}$ polyvinylidene difluoride filter before HPLC analysis.

\section{High-performance liquid chromatography conditions}

The analysis was performed using a RStech HECTOR-M C18 column $(250 \times 4.6 \mathrm{~mm}, 5 \mu \mathrm{m}$; Daejeon, Korea $)$, with a Shimadzu HPLC 20A Prominence series (Kyoto, Kyoto, Japan) instrument that consisted of an LC-20 AD liquid chromatography unit, SPD-20A UV/VIS detector, SPD-M20A DAD, SIL-20A auto sampler, and CBM-20A communication bus module. Water and various organic solvents were used in the mobile phase. A flow rate of 1 $\mathrm{mL} / \mathrm{min}$ and an injection volume of $10 \mu \mathrm{L}$ were applied. The detection wavelength was $210 \mathrm{~nm}$ and the total analytical time was $30 \mathrm{~min}$.

\section{Optimization of analytical sample preparation}

The effect of the solvent type, extraction process, and extraction time were verified to achieve efficient extraction for analytical sample preparation. Acetone, chloroform, dichloromethane, ethyl acetate, and hexane were verified for their use as an optimal extraction solvent. Each $20 \mathrm{~mL}$ of solvent was added to a $0.2-\mathrm{g}$ sample. To determine the optimal extraction solvent, sonication and reflux were compared to optimize the extraction process at $30 \mathrm{~min}$. Various extraction times $(10,30,60,90$, and $120 \mathrm{~min}$ ) were also investigated to determine the optimal extraction time.

\section{Method validation}

The new method was validated by selectivity, linearity, intra- and inter-day precision and accuracy, limit of detection (LOD), and limit of quantification (LOQ) according to the International Council for Harmonisation (ICH) guidelines (ICH 2009) and the Korean Ministry of Food and Drug Safety (MFDS) guidelines (MFDS 2014). The selectivity was confirmed by a comparison of retention time and UV absorption spectra with standards and samples. Linearity was evaluated by the coefficient of determination $\left(R^{2}\right)$ of a calibration curve in the inotodiol range of $12.5-200 \mu \mathrm{g} / \mathrm{mL}$ and $3 \beta$-hydroxylanosta-8,24dien-21-al range of $25-400 \mu \mathrm{g} / \mathrm{mL}$. Intra- and inter-day precision and accuracy were verified by analyzing three concentrations of standard solutions (inotodiol, 25, 50, and $100 \mu \mathrm{g} / \mathrm{mL} ; 3 \beta$-hydroxylanosta-8,24-dien-21-al, 50, 100 , and $200 \mu \mathrm{g} / \mathrm{mL}$ ) five times per day on five consecutive days. Intra- and inter-day variation for precision was expressed as the relative standard deviation (RSD). Intraand inter-day accuracy was verified by spiking a standard solution into a sample, and then calculated as percentages. The LOD and LOQ were estimated as three and ten times the signal-to-noise ratio, respectively.

\section{Application of the developed method for quality control} Various mushroom species, such as Auricularia auriculajudae, Ganoderma lucidum, Lentinula edodes, Phellinus linteus, and Inonotus hispidus, were analyzed with the HPLC method using the two proposed marker compounds to evaluate their suitability for the discrimination of I. obliquus and analytical quality control.

\section{Statistical analysis}

The experimental data were expressed as the mean and RSD. The content criteria of the marker compounds for I. obliquus were calculated using the RSD and linear regression methods (Kang et al. 2012). For the RSD method, a Shapiro-Wilk normality test was performed at the level of $p=0.05$; the null hypothesis was that the quantitative data followed a normal distribution. When data were normally distributed, the RSD method was used by applying the following equation: mean $-2.398 \times \mathrm{S} /$ $\sqrt{\mathrm{n}}$ (Standard deviation/the number of sample), to produce a test statistic corresponding to $-1 \%$ of the total probability based on the mean from a $t$ distribution. The content criteria of the marker compounds were also estimated by linear regression. The established criteria were applied to mushrooms to verify their suitability for quality control considering the fail sample number. The content criteria were established by a statistical analysis using $R$ program and R Studio (Freeware version 3.5.0). 


\section{Results and discussion}

\section{High-performance liquid chromatography conditions}

In the detection wavelength screening of the two proposed marker compounds using water-acetonitrile as the mobile phase, the maximum absorption $\left(\lambda_{\max }\right)$ was less than $190 \mathrm{~nm}$ for both compounds. However, this was not the correct detection wavelength, and the baseline and UV cut-off of the mobile phase were unstable; therefore, $210 \mathrm{~nm}$ was selected as the detection wavelength. To achieve an efficient separation of the two marker compounds, various elution conditions were assessed. Among the elution conditions, isocratic elution using 95\% acetonitrile was selected as the optimal mobile phase condition based on the shorter running time, higher repeatability, better separation, and tailing factor, because the polarity of the two marker compounds was low (Fig. 2).

\section{Optimization of the extraction method}

The extraction efficiency of some compounds is affected by solvent polarity. The chemical structures of inotodiol and 3 3 -hydroxylanosta-8,24-dien-21-al are similar to that of cholesterol; therefore, the two markers might be efficiently extracted in low-polarity solvents (Zheng et al. 2011). To verify this hypothesis, various low-polarity solvents were examined to improve the extraction efficiency of the two markers. The polarity of extraction solvents decreased in the order of acetone, ethyl acetate, chloroform, dichloromethane, and hexane. In general, hexane, chloroform, and dichloromethane have high extraction efficiencies for cholesterol (Bligh and Dyer 1959; Folch et al. 1957; McDonald et al. 2012). The polarities of the two markers are higher than that of cholesterol because they contain more hydroxyl and aldehyde groups. Therefore, it was presumed that a more polar solvent than hexane, such as chloroform or dichloromethane, could be the optimal extraction solvent for the two marker compounds (Albuquerque et al. 2016). In the experimental analysis, chloroform was selected as the extraction solvent for both marker compounds (Figure S3). Sonication had a higher extraction efficiency than reflux over a 30-min period (Figure S4). The

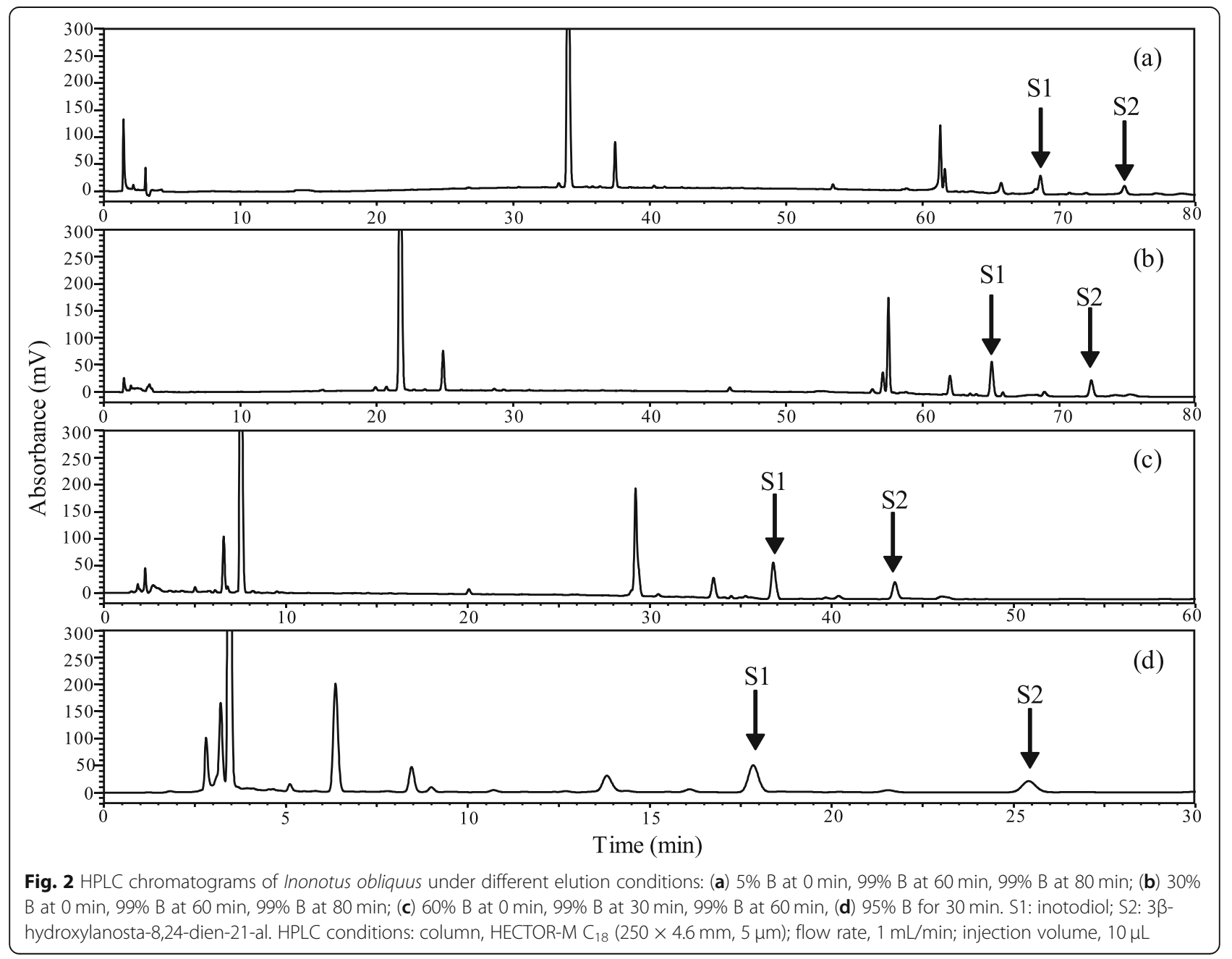


optimal extraction time was 30 min (Figure S5), while an excessive processing time reduced the extraction yield of the two markers, as also noted in other studies (Wang and Weller 2006).

\section{Method validation}

The selectivity of a method indicates its ability to quantify a target compound within a matrix of other substances that could potentially interfere with the analysis of complex samples (Andrade et al. 2016). The retention times of inotodiol and 3 $\beta$-hydroxylanosta-8,24-dien-21al in samples matched with the standards at 17.9 and 25.4 min, respectively. The UV spectra of inotodiol and 3ß-hydroxylanosta-8,24-dien-21-al were also the same as their standard compounds (Figure S6). Consequently, two peaks in the samples were confirmed as inotodiol and 3ß-hydroxylanosta-8,24-dien-21-al. The peak resolution of each marker compound was greater than 1.5, indicating a good separation without interference from other peaks. The $R^{2}$ of the calibration curve within the target range was greater than 0.9997 for both marker compounds, which confirmed that the linear regression method was appropriate (Figure S7). The LOD and LOQ of inotodiol were 0.07 and $0.24 \mu \mathrm{g} / \mathrm{mL}$, respectively, while the corresponding values for $3 \beta$-hydroxylanosta-8, 24-dien-21-al were calculated as 0.4 and $1.4 \mu \mathrm{g} / \mathrm{mL}$, respectively (Table S2). For both marker compounds, the intra-day precision was below $2.5 \%$ and inter-day precision was less than $4.0 \%$, and the intra-day accuracy was 96.7 to $104.6 \%$ and inter-day accuracy was 98.6 to $104.9 \%$ (Table S3). The precision and accuracy of the developed method were less than 5\% RSD, which confirmed the suitability of this method according to the method validation guidelines of the MFDS (Korea) and the ICH method validation guidelines. The recoveries for both marker compounds ranged from 93.9 to $114.4 \%$, with less than 1\% RSD (Table S4). The HPLC method was considered to be suitable for the qualification and quantitation of inotodiol and $3 \beta-$ hydroxylanosta-8,24-dien-21-al and could be used for the quality control of I. obliquus.

\section{Application of the developed method to mushrooms}

A chromatographic pattern analysis was performed to discriminate I. obliquus from other mushroom species because the appearance of extracts obtained from other mushrooms and raw mushrooms was similar to the extracts of I. obliquus and raw I. obliquus. It was important to analyze I. obliquus alongside other mushrooms of the same genus. Therefore, one mushroom of the same genus (I. hispidus) and four mushrooms of a different genus (A. auricula-judae, G. lucidum, L. edodes, and P. linteus) were analyzed and the results were compared to those obtained for I. obliquus (Fig. 3).
In the pattern analysis results to select marker compounds, six peaks were observed before a retention time of $10 \mathrm{~min}$ for all mushrooms, and a peak at 14 min for I. obliquus seemed to be suitable marker, but other unknown small peaks were observed at the same retention time for G. lucidum, L. edodes, and $P$. linteus. However, only for I. obliquus were both inotodiol and 3 $\beta$-hydroxylanosta-8,24-dien-21-al detected. This result indicates that the detection of both inotodiol and 3 $\beta$-hydroxylanosta-8,24-dien-21-al can be used to discriminate $I$. obliquus from mushrooms of the same or different genera, confirming the suitability of these compounds as new unique and representative marker compounds for the quality control of $I$. obliquus.

Ten samples of I. obliquus mushrooms purchased at a market were compared by pattern analysis, and the chromatographic pattern of the mushrooms was the same. The inotodiol and 33-hydroxylanosta-8,24-dien-21-al concentrations in mushrooms were $0.625 \sim 2.399$ and $1.357 \sim 2.553 \mathrm{mg} / \mathrm{g}$, respectively (Table 1 ).

For the quality control, the content criteria of the two marker compounds established for I. obliquus mushrooms were determined using two statistical methods: RSD and linear regression. The content criteria calculated by the two methods were different, with the number of failed samples shown in Table 2 . For the RSD method, a Shapiro-Wilk normality test was initially performed and the $p$ values of inotodiol and 3ß-hydroxylanosta-8,24-dien-21-al were 0.7984 and 0.8065 . The null hypothesis was that the quantitative data of collected samples would have a normal distribution. However, because fewer than 30 samples were used, the samples followed a Student $t$ distribution. The content criteria calculated by the RSD method were $1.165 \mathrm{mg} / \mathrm{g}$ for inotodiol and $1.717 \mathrm{mg} / \mathrm{g}$ for 3ß-hydroxylanosta-8,24-dien-21-al. For the linear regression method, the regression equation was as follows: $y=0.7244+0.1445 \times(x=1 \sim 10)$, with an adjusted $R^{2}$ of 0.8622 , for inotodiol and $y=1.4219+$ $0.09692 \times(x=1 \sim 10)$, with an adjusted $R^{2}$ of 0.8594 , for 3 $\beta$-hydroxylanosta-8,24-dien-21-al. The content criteria estimated by the linear regression method was $0.869 \mathrm{mg} / \mathrm{g}$ for inotodiol and $1.519 \mathrm{mg} / \mathrm{g}$ for $3 \beta$ hydroxylanosta-8,24-dien-21-al. The failed sample number based on the new content criteria differed slightly depending on the statistical method used for the content criteria, with two samples failing for the RSD method and one sample failing for the linear regression method. According to Kim et al. (2015), the RSD method is more suitable to determine the content criteria when data are normally distributed; otherwise, the linear regression method should be used. In this study, the data were normally 


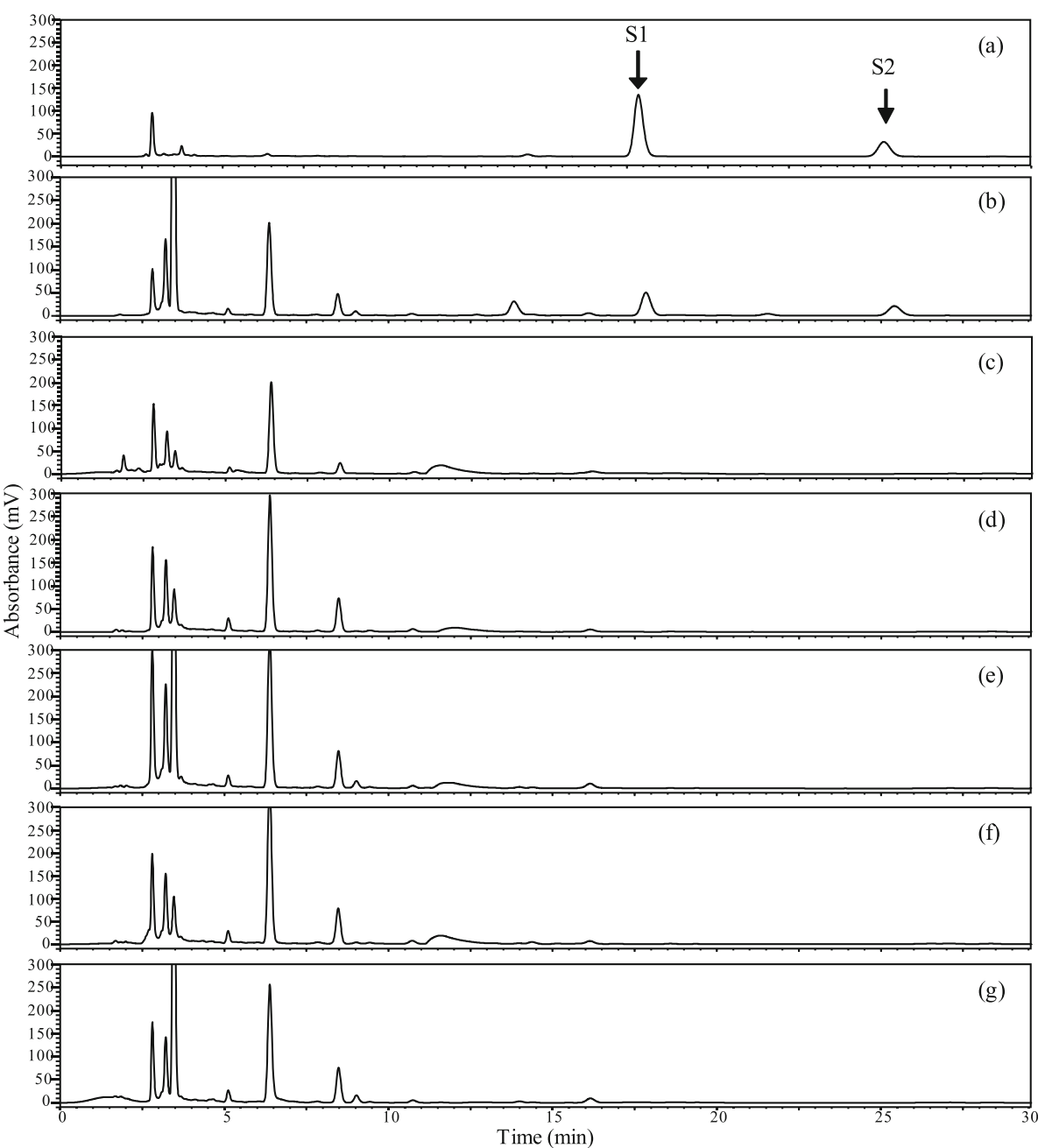

Fig. 3 Chromatographic pattern analysis of (a) a mixture of standards, (b) Inonotus obliquus, (c) Inonotus hispidus, (d) Auricularia auricula-judae, (e) Ganoderma lucidum, (f) Lentinula edodes, (g) Phellinus linteus. S1: inotodiol; S2: 3ß-hydroxylanosta-8,24-dien-21-al. HPLC conditions: column, HECTOR-M $\mathrm{C}_{18}(250 \times 4.6 \mathrm{~mm}, 5 \mu \mathrm{m})$; eluent, $95 \%$ acetonitrile; flow rate, $1 \mathrm{~mL} / \mathrm{min}$; injection volume, $10 \mu \mathrm{L}$

Table 1 Quantitative analysis of two marker compounds in I. obliquus mushrooms and its commercial products $(n=3)$

\begin{tabular}{llll}
\hline Sample & No. & \multicolumn{3}{l}{ Content $(\mathbf{m g} \mathbf{g})$} \\
\cline { 3 - 4 } & & Inotodiol & 3ß-hydroxylanosta-8,24-dien-21-al \\
\hline Mushrooms & 1 & $1.276 \pm 0.198$ & $1.888 \pm 0.198$ \\
& 2 & $2.399 \pm 0.100$ & $2.004 \pm 0.100$ \\
3 & $1.680 \pm 0.034$ & $1.763 \pm 0.034$ \\
4 & $1.407 \pm 0.024$ & $1.950 \pm 0.024$ \\
5 & $1.556 \pm 0.004$ & $1.984 \pm 0.004$ \\
6 & $0.625 \pm 0.064$ & $1.357 \pm 0.064$ \\
7 & $1.116 \pm 0.045$ & $2.081 \pm 0.045$ \\
8 & $1.616 \pm 0.148$ & $2.553 \pm 0.148$ \\
9 & $1.804 \pm 0.247$ & $2.217 \pm 0.247$ \\
10 & $1.712 \pm 0.166$ & $1.752 \pm 0.165$
\end{tabular}

N.D. not detected distributed. Thus, the recommended content criteria for I. obliquus were $1.165 \mathrm{mg} / \mathrm{g}$ for inotodiol and $1.717 \mathrm{mg} / \mathrm{g}$ for 33-hydroxylanosta-8,24-dien-21-al using the RSD method. This study established criteria using Student $t$ distribution for the RSD method because the sample size is less than 30. To confirm the more accurate content criteria, a larger number of I. obliquus samples should be analyzed in a future study.

\section{Conclusion}

Inonotus obliquus is widely used as a functional food. However, the use of chromogen complex, which has long been used as a marker compound for quality control, yields ambiguous results and makes precise quality control difficult. The aim of this study was to develop an analytical method using new marker compounds for the quality control of I. obliquus mushrooms by HPLC. 
Table 2 Content criteria of I. obliquus mushroom depending on statistical methods $(n=10)$

\begin{tabular}{|c|c|c|c|c|}
\hline \multirow{2}{*}{$\begin{array}{l}\text { Statistical process } \\
\text { Marker compounds }\end{array}$} & \multicolumn{2}{|c|}{ RSD method } & \multicolumn{2}{|c|}{ Linear regression method } \\
\hline & Inotodiol & 3ß-Hydroxylanosta-8,24-dien-21-al & Inotodiol & $3 \beta$-Hydroxylanosta-8,24-dien-21-al \\
\hline Lower content criteria (mg/g) & 1.165 & 1.717 & 0.869 & 1.519 \\
\hline Fail sample number & 2 & 1 & 1 & 1 \\
\hline
\end{tabular}

Inotodiol and 3 $\beta$-hydroxylanosta-8,24-dien-21-al were selected as new marker compounds for I. obliquus because of their uniqueness and bioactivity. A new HPLC method was developed in accordance with the method validation guidelines of the MFDS and ICH. The new method could completely discriminate I. obliquus from those of mushrooms of the same genus and with a similar morphology. For the quantitative monitoring of $I$. obliquus samples, the recommended content criteria were $1.165 \mathrm{mg} / \mathrm{g}$ for inotodiol and $1.717 \mathrm{mg} / \mathrm{g}$ for $3 \beta$ hydroxylanosta-8,24-dien-21-al using the RSD method. Consequently, the new HPLC method is promising not only for the discrimination but also for quality control of I. obliquus.

\section{Supplementary Information}

The online version contains supplementary material available at https://doi. org/10.1186/s40543-020-00249-z.

Additional file 1: Table S2. Sample information for analysis. Table S2. LOD and LOQ of marker compounds. Table S3. Intra-/inter-day precision and accuracy. Table S4. Recovery of marker compounds $(n=3)$.

Additional file 2: Figure S1. Morphologies of (a) I. obliquus mushroom, (b) commercial I. obliquus product, (c) A. auricula-judae mushroom, (d) G. lucidum mushroom, (e) L edodes mushroom, (f) P. linteus mushroom and (g) I. hispidus mushroom. Figure S2. Chromatograms of (a) blank, (b) inotodiol and (c) 3ß-hydroxylanosta-8,24-dien-21-al. HPLC conditions: Column; HECTOR-M C 18 (250 x 4.6 mm, $5 \mu \mathrm{m})$, eluent; $99 \%$ acetonitrile, flow rate of $1 \mathrm{~mL} / \mathrm{min}$, injection volume of $10 \mu \mathrm{L}$. Figure $\mathbf{S 3}$. Extraction efficiency compounds depending on various solvents. Figure S4. Extraction efficiency of marker depending on extraction method. Figure S5. Extraction efficiency of marker compounds depending on extraction time by sonication. Figure S6. Comparison of UV spectra between sample and standards: (a) inotodiol and (b) 3ß-hydroxylanosta-8,24-dien-21-al. Figure

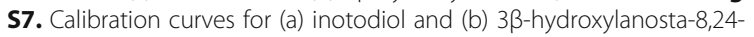
dien-21-al.

\begin{abstract}
Abbreviations
HPLC: High-performance liquid chromatography; I. obliquus: Inonotus obliquus; UV: Ultraviolet; MFDS: Korean Ministry of Food and Drug Safety; ICH: International Council for Harmonisation; LOD: Limit of detection; LOQ: Limit of quantification; $\mathrm{S} / \sqrt{\mathrm{n}}$ : Standard deviation/the number of sample; I. hispidus: Inonotus hispidus; A. auricula-judae: Auricularia auricu-lajudae; G. lucidum: Ganoderma lucidum; L. edodes: Lentinula edodes; P. linteus: Phellinus linteus
\end{abstract}

\section{Acknowledgements}

The authors thank Institute of Drug Research and Development in College of Pharmacy, CNU.

\section{Authors' contributions}

Conceptualization: JS KANG, KT KIM

Writing: JH KIM

Review: IS KANG, KT KIM

Analysis: SK PARK, JH KIM, JW CHO
Isolation and identification: BV LE, YH KIM, H BAO

Additional works: JH KIM

All authors agreed position and publication

\section{Funding}

Chungnam National University (CNU) Research Grant (2016-1531-01), a grant for Institute of Drug Research and Development in College of Pharmacy (CNU) (National Research Foundation of Korea), and a grant from Korea Food Research Institute (E0121304-05).

Availability of data and materials

The datasets of this manuscript are available upon request.

\section{Competing interests}

The authors declare no conflict of interest.

\section{Author details}

${ }^{1}$ College of Pharmacy, Chungnam National University, Daejeon 34134, Korea. ${ }^{2}$ Institute of Marine Biochemistry (IMBC), Vietnam Academy of Science and Technology (VAST), Hanoi, Vietnam. ${ }^{3}$ College of Chinese Medicinal Materials, Jilin Agricultural University, Changchun 130118, China. ${ }^{4}$ Department of Food Science and Technology, Dong-Eui University, Busan 47340, Korea.

Received: 29 June 2020 Accepted: 10 November 2020

Published online: 05 December 2020

\section{References}

Aguiar PH, Tsanaclis AM, Tella OI Jr, Plese JP. Proliferation rate of intracranial meningiomas as defined by the monoclonal antibody MIB-1: correlation with peritumoural oedema and other clinicoradiological and histological characteristics. Neurosurg. Rev. 2003;26(3):221-8.

Albuquerque TG, Oliveira MB, Sanches-Silva A, Costa HS. Cholesterol determination in foods: comparison between high performance and ultrahigh performance liquid chromatography. Food Chem. 2016;193:18-25.

Andrade JK, Komatsu E, Perreault H, Torres YR, da Rosa MR, Felsner ML. In house validation from direct determination of 5-hydroxymethyl-2-furfural (HMF) in Brazilian corn and cane syrups samples by HPLC-UV. Food Chem. 2016;190: 481-6.

Bach F, Helm CV, Bellettini MB, Maciel GM, Haminiuk CWI. Edible mushrooms: a potential source of essential amino acids, glucans and minerals. Int. J. Food Sci. Technol. 2017;52(11):2382-92.

Bligh EG, Dyer WJ. A rapid method of total lipid extraction and purification. Can. J. Biochem. Physiol. 1959;37(8):911-7.

Chung MJ, Chung CK, Jeong Y, Ham SS. Anticancer activity of subfractions containing pure compounds of Chaga mushroom (Inonotus obliquus) extract in human cancer cells and in Balbc/c mice bearing Sarcoma-180 cells. Nutr. Res. Pract. 2010;4(3):177-82.

Davidson J. The chromogen method for determining the digestibility of dried grass by sheep. J. Sci. Food Agric. 1954;5(5):209-12.

Du D, Zhu F, Chen X, Ju X, Feng Y, Qi LW, Jiang J. Rapid isolation and purification of inotodiol and trametenolic acid from Inonotus obliquus by high-speed counter-current chromatography with evaporative light scatting detection. Phytochem. Anal. 2011;22(5):419-23.

Folch J, Lees M, Stanley GHS. A simple method for the isolation and purification of total lipides from animal tissues. J. Biol. Chem. 1957;226(1):497-509.

ICH. (2009). Validation of analytical procedures: text and methodology Q2(R1).

Ju HK, Chung HW, Hong SS, Park JH, Lee JM, Kwon SW. Effect of steam treatment on soluble phenolic content and antioxidant activity of the Chaga mushroom (Inonotus obliquus). Food Chem. 2010;119(2):619-25.

Kang JS, Jang YS, Baek KO. Establish of concentration limit of marker compound in herbal drug. Korea Patent 10-1123081 Feb 2012. 
Kim KT, Shin MC, Kim HH, Cho CW, Lee WJ, Woo ER, Kim KH, Kang JS. Specification and analysis of multiple marker compounds for quality control of Mori cortex radicis by HPLC. Bull. Korean Chem. Soc. 2015;36(1):117-22.

Le VNH, Lee WJ, Kim YH, Chae GH, Chin YW, Kim KT, Kang JS. High-performance liquid chromatography method development for the quality control of Ginkgonis Semen. Arab. J. Chem. 2017;10(6):792-800.

McDonald JG, Smith DD, Stiles AR, Russell DW. A comprehensive method for extraction and quantitative analysis of sterols and secosteroids from human plasma. J. Lipid Res. 2012;53(7):1399-409.

Medizina. The State Pharmacopoeia of USSR, vol. 2. Russia: Pharmacological committee of ministry of Health of the USSR; 1990.

MFDS. Year book of imported food inspection. Korea: Ministry of Food and Drug Administration; 2013

MFDS. Korean Pharmacopoeia. Korea: Ministry of Food and Drug Administration; 2014.

MFDS. Year book of imported food inspection. Korea: Ministry of Food and Drug Administration; 2017.

Moon BH, Lee WC. Studies on the anti-cancer activity of Chaga mushroom Extract. J. Korean Med. 2009;30(4):1-12.

Nakata T, Yamada T, Taji S, Ohishi H, Wada S, Tokuda H, Sakuma K, Tanaka R. Structure determination of inonotsuoxides $\mathrm{A}$ and $\mathrm{B}$ and in vivo anti-tumor promoting activity of inotodiol from the sclerotia of Inonotus obliquus. Biorg. Med. Chem. 2007;15(1):257-64.

Nguyet TMN, Lomunova M, Le BV, Lee JS, Park SK, Kang JS, Kim YH, Hwang I. The mast cell stabilizing activity of Chaga mushroom critical for its therapeutic effect on food allergy is derived from inotodiol. Int. Immunopharmacol. 2018; 54:286-95.

Oxford University Press. (2010). Oxford English Dictionary: Oxford University Press.

Panarelli NC, Yantiss RK, Yeh MM, Liu Y, Chen Y-T. Tissue-specific cadherin CDH17 is a useful marker of gastrointestinal adenocarcinomas with higher sensitivity than CDX2. Am. J. Clin. Pathol. 2012;138(2):211-22.

Shikov AN, Pozharitskaya ON, Makarov VG, Wagner H, Verpoorte R, Heinrich M. Medicinal plants of the Russian Pharmacopoeia; their history and applications. J. Ethnopharmacol. 2014;154(3):481-536.

Sun JE, Ao ZH, Lu ZM, Xu HY, Zhang XM, Dou WF, Xu ZH. Antihyperglycemic and antilipidperoxidative effects of dry matter of culture broth of Inonotus obliquus in submerged culture on normal and alloxan-diabetes mice. J. Ethnopharmacol. 2008;118(1):7-13.

Tian J, Hu X, Liu D, Wu H, Qu L. Identification of Inonotus obliquus polysaccharide with broad-spectrum antiviral activity against multi-feline viruses. Int. J. Biol. Macromol. 2017;95:160-7.

Wang C, Chen Z, Pan Y, Gao X, Chen H. Anti-diabetic effects of Inonotus obliquus polysaccharides-chromium (III) complex in type 2 diabetic mice and its subacute toxicity evaluation in normal mice. Food Chem. Toxicol. 2017a;108(Pt B):498-509.

Wang J, Hu W, Li L, Huang X, Liu Y, Wang D, Teng L. Antidiabetic activities of polysaccharides separated from Inonotus obliquus via the modulation of oxidative stress in mice with streptozotocin-induced diabetes. PLoS One. 2017b;12(6):1-19.

Wang L, Weller CL. Recent advances in extraction of nutraceuticals from plants. Trends Food Sci. Technol. 2006;17(6):300-12.

Xu XQ, Zhang X, Chen C. Stimulated production of triterpenoids of Inonotus obliquus using methyl jasmonate and fatty acids. Ind. Crops Prod. 2016;85: 49-57.

Yan J, Han Z, Qu Y, Yao C, Shen D, Tai G, Cheng H, Zhou Y. Structure elucidation and immunomodulatory activity of a beta-glucan derived from the fruiting bodies of Amillariella mellea. Food Chem. 2018;240:534-43.

Zheng W, Miao K, Liu Y, Zhao Y, Zhang M, Pan S, Dai Y. Chemical diversity of biologically active metabolites in the sclerotia of Inonotus obliquus and submerged culture strategies for up-regulating their production. Appl. Microbiol. Biotechnol. 2010;87(4):1237-54.

Zheng W. Zhang M, Zhao Y, Miao K. Pan S, Cao F. Dai Y. Analysis of antioxidant metabolites by solvent extraction from sclerotia of Inonotus obliquus (Chaga). Phytochem. Anal. 2011;22(2):95-102.

\section{Publisher's Note}

Springer Nature remains neutral with regard to jurisdictional claims in published maps and institutional affiliations.

\section{Submit your manuscript to a SpringerOpen ${ }^{\circ}$ journal and benefit from:}

- Convenient online submission

- Rigorous peer review

- Open access: articles freely available online

- High visibility within the field

- Retaining the copyright to your article

Submit your next manuscript at $\boldsymbol{\nabla}$ springeropen.com 